section presenting nothing abnormal. Under the microscope its capillaries were filled with white corpuscles, which seemed to outnumber the red ones. Glisson's capsule was extensively infiltrated with round cells. The mesenteric glands in general were enlarged, those on the posterior wall of the circumscribed peritoneal cavity projecting as nodular masses of the size of plums, red, and resembling the spleen in appearance.

\title{
A CASE OF IDIOPATHIC GLOSSITIS.
}

BY URANUS O. B. WiNgate, M. D., OF WEllegley.

Ars three P. M., June 9, 1876, I was called to Katie-M., a domestic, single, born in Ireland, who had been in this country two weeks, was twenty years of age, had always been healthy and robust, and stated that she never took any medicine in her life. On the afternoon of the day before, she began to have a pain at the base of her tongue. She passed a restless night, but attended to her work in the morning until about noon, when she grew rapidly worse and was obliged to give up and go to berl.

I found her complaining of great pain in her tongue. She had romited several times, the vomitus consisting of food only. She stated that she felt well with the exception of the pain in her tongue, which was much swollen, hard, and covered with a yellowish-white coat. On attempting to protrude it, she suffered great pain, and any movement of the organ seemed almost impossible. She spoke with great difficulty, and deglutition was extremely painful. Pulse, 130 per minute. Temperature in the axilla, $103.2^{\circ} \mathrm{F}$. There was a slight flow of saliva, and moderate thirst. The patient presented a neat appearance, and the hygienic surroundings were good. Hot applications directly under the chin, chlorate of potash wash for the mouth every half-hour, and bits of ice on the tongue all the time when awake were ordered. Also fifteen grains of bromide of potassium with one fifth of a grain of morphine were to be given in a wine-glass of water every four hours, to relieve pain and produce sleep. Milk diet.

June 10th. The patient slept a little during the night, while under the influence of morphia. The tongue was swollen so badly that she could not speak intelligibly, and was very painful. She had not vomited for twenty hours. The bowels were constipated. There was no pain except in the tongne. The tonsils were unaffected. Pulse, 120. Temperature, $103^{\circ}$. There was slight dyspnœa. The same treatment was continued, and in addition pil. hydrarg. grs. iii. at night were ordered, to be followed early in the morning by a half-ounce of sulphate of magnesia in solution.

June 11th. She had slept very little. The tongue was still swollen 
badly. The coating had disappeared, and the tongue looked clean and presented a very dark red color. It filled the mouth and protruded slightly between the teeth. There was a little flow of saliva; less pain, and she could swallow milk much better. The patient now complained of weakness. There was complete anorexia. Pulse, 90. Temperature, $100^{\circ}$. The same treatment was continued. In addition to milk, beef-tea and eggnog were ordered.

June 12th. The patient was much better. She had slept well all night. The tongue was very red, but less painful and considerably reduced in size. She could talk quite well, and could swallow food and medicine with much greater ease. Pulse, 80. Temperature, 98.5 . She complained only of weakness and loss of appetite. She was to continue the mouth wash, to use the ice occasionally, to omit all other medicine, and to have two teaspoonfuls of Wyeth's elixir of calisaya three times daily.

June 15th. She had returned to her work, and stated that she felt quite well. The tongue was a little enlarged, and still looked abnormally red.

June 20th. The tongue looked natural, and she had made a complete recovery.

George B. Wood, M. D., in his Treatise on the Practice of Medicine, says, "Glossitis may result from the usual causes of inflammation. Perhaps the most frequent cause is direct injury, resulting from irritating or corrosive substances, scalding drinks, wounds or bruises, or the bites or stings of venomous insects. Sometimes the affection is produced by a direct propagation of inflammation of the tonsils, and it occasionally arises in the course of exanthematous fevers. In some instances the chief force of mercurial action appears to fall upon the tongue and produce tumefaction." The above case could not be traced to any of these causes. Flint, in his work on Theory and Practice makes no mention of glossitis as a disease per se. Dacosta speaks of it as a dangerous affection, and fortunately rare. Tanner in his Practice of Medicine says, "Inflammation of the tongue is a rare affection now that mercury is seldom used so as to produce salivation. It is generally met with as an accompaniment of other diseases rather than as an idiopathic affection." Fartlier on he says, "When glossitis arises idiopathically, it gives rise to fever, mental depression, and general weakness," thus indicating that it may be idiopathic. Erichsen, Druitt, Bryant, and others speak of glossitis as a rare affection.

In the Transactions of the Medical Society of the College of Physicians, published in the Dublin Journal of Medical Science for April, 1876, can be found an account of a case of idiopathic glossitis, by Henry G. Croly, F. R. C. S. I. ${ }^{1}$ Mr. Croly, it appears, has observed thirteen or

1 Jounna I, July 6, 1876, page 23. 
fourteen cases of glossitis during twenty years of hospital practice, and he thinks it not so rare as some have supposed. He believes that the inflammation commences at the base and extends forward. This seems of some interest, as most of the books speak of it as commencing at the tip and extending back. In the above case it commenced at the base, and gradually extended over the entire organ. In Mr. Croly's last case one side of the organ only was affected.

As regards treatment, $\mathrm{Mr}$. Croly recommends purgatives and free incisions, and he gives some useful and important suggestions in regard to making the latter. Undoubtedly free incisions give great relief. They were proposed in this case, but were refused, and as many patients fear the knife more than almost any risk, the question arises as to whether or not most patients would not do quite as well without the knife as with it, provided that other treatment is not neglected. The rareness of reported cases of idliopathic glossitis in the journals has prompted me to report this case.

\section{CASE OF A'TRESIA ANI VAGINALIS.'}

BY HeNry TUCK, M. D. (HARv.), Visiting Physician to the Buston Lying.In Hospital.2

A. B., single, aged twenty-six, of American parentage, a resident of a neighboring town, was sent to Boston by a medical friend to be under my care in her confinement. She was a lady in good social position, but while riding with a young man, her intended husband, having been rendered unconscious by drugged candy, as she claims, was raped by him. This occurred on May 26th. In the following September, not having seen her catamenia for three months, and finding herself increasing in size, she consulted her family physician, who, not suspecting the nature of the case, treated her for amenorrlicea. Pregnancy was finally suspected, and she was treated by an irregular physician, who tried to bring on an abortion by the use of drugs. No operation, however, was attempted. After this she passed into the hands of a competent physician, who made a vaginal examination, and ascertained the real state of the case. By him she was a few months later sent to Boston and placed under my care. Confinement was expected about March 3d but did not occur till March 14th, making her period of gestation two hundred and ninety-three days. The head presented and the labor was normal in every way, except that it was rather long, twenty-three hours, and there was very little liquor amnii, as has been before noticed in cases of prolonged gestation. The

1 Foerster's Path. Anat., part ii., p. 98.

2 Read before the Obstctrical Society of Boston. 